\title{
Quantitative Clinical Nuclear Cardiology, Part 1: Established Applications
}

\author{
Ernest V. Garcia ${ }^{1}$, Piotr Slomka ${ }^{2}$, Jonathan B. Moody ${ }^{3}$, Guido Germano ${ }^{2}$, and Edward P. Ficaro ${ }^{3,4}$ \\ ${ }^{1}$ Department of Radiology and Imaging Sciences, Emory University, Atlanta, Georgia; ${ }^{2}$ Department of Medicine, Cedars-Sinai \\ Medical Center, Los Angeles, California; ${ }^{3}$ INVIA Medical Imaging Solutions, Ann Arbor, Michigan; and ${ }^{4}$ Division of Cardiovascular \\ Medicine, Department of Internal Medicine, University of Michigan, Ann Arbor, Michigan
}

\begin{abstract}
Learning Objectives: On successful completion of this activity, participants should be able to (1) know the basics of how quantitative parameters of relative left ventricular myocardial perfusion are measured and used clinically to differentiate patients with normal versus abnormal distributions; (2) know the basics of how quantitative parameters of left ventricular myocardial global and regional function is measured and used clinically to differentiate patients with normal versus abnormal function; and (3) describe and know how to use clinically nonperfusion quantitative parameters obtained from perfusion studies to modify the likelihood of the presence and extent of coronary artery disease in patients.
\end{abstract}

Financial Disclosure: Dr. Moody is an employee of Invia. Dr. Slomka receives grants from the National Institutes of Health and Siemens Medical Systems and receives software royalties from Cedars-Sinai. Dr. Germano receives royalties from most nuclear medicine companies. Dr. Ficaro is an employee of Invia. Dr. Garcia receives grants from Syntermed and GE Healthcare and receives royalties from Syntermed. The authors of this article have indicated no other relevant relationships that could be perceived as a real or apparent conflict of interest.

CME Credit: SNMMI is accredited by the Accreditation Council for Continuing Medical Education (ACCME) to sponsor continuing education for physicians. SNMMI designates each JNM continuing education article for a maximum of 2.0 AMA PRA Category 1 Credits. Physicians should claim only credit commensurate with the extent of their participation in the activity. For CE credit, SAM, and other credit types, participants can access this activity through the SNMMI website (http://www.snmmilearningcenter.org) through November 2022.

SPECT myocardial perfusion imaging has attained widespread clinical acceptance as a standard of care for patients with known or suspected coronary artery disease. A significant contribution to this success has been the use of computer techniques to provide objective quantitative assessment in the standardization of the interpretation of these studies. Software platforms have been developed as a pipeline to provide the quantitative algorithms researched, developed and validated to be clinically useful so diagnosticians everywhere can benefit from these tools. The goal of this continuing medical education article (part 1 ) is to describe the many quantitative tools that are clinically established and, more importantly, how clinicians should use them routinely in interpretation, clinical management, and therapy guidance for patients with coronary artery disease.

Key Words: quantitative LV perfusion; quantitative LV function; ischemic burden; myocardial perfusion imaging; transient ischemic dilation; summed stress score

J Nucl Med 2019; 60:1507-1516

DOI: 10.2967/jnumed.119.229799

$\mathbf{N}$ perfusion, right and left ventricular (LV) function, synchrony of contraction, myocardial substrate metabolism, innervation, and inflammation. Nuclear cardiology is inherently digital and quantitative. The

Received Apr. 12, 2019; revision accepted Jul. 11, 2019.

For correspondence or reprints contact: Ernest V. Garcia, Department of Radiology and Imaging Sciences, 101 Woodruff Circle, Room 1203, Emory University, Atlanta, GA 30322.

E-mail: ernest.garcia@emory.edu

This article is being jointly published in The Journal of Nuclear Medicine (DOI: 10.2967/jnumed.119.229799) and the Journal of Nuclear Cardiology (https://doi.org/10.1007/s12350-019-01906-6).

COPYRIGHT (c) 2019 Society of Nuclear Medicine and Molecular Imaging \& American Society of Nuclear Cardiology. images are easily transformed into a digital pixel array. The scientific and clinical foundation of nuclear cardiology is also squarely framed in an extensive evidence base originating from the many books and thousands of articles published over the last 40 years (1). SPECT and PET imaging guidelines have also been published that describe the clinically established applications many of the quantitative parameters discussed $(2,3)$.

This work, divided into 2 articles, is meant to be a snapshot in time of the state of the field of quantitative nuclear cardiology today. The purpose of the authors is 2-fold. In part 1, the goal is to describe the many quantitative tools that are clinically established. In part 2, the goal is to describe the tools that are evolving or emerging in nuclear cardiology. The secondary-albeit the most important-goal is for diagnosticians to use the established tools to improve the management of patients with heart disease and to learn about and anticipate the use of the evolving and emerging tools.

\section{ESTABLISHED CLINICAL APPLICATIONS}

\section{Database Relative Myocardial Perfusion Imaging (MPI) Quantification}

Perfusion Quantification. Quantification of myocardial perfusion extracted from the LV myocardium is one of the base modules incorporated into nuclear cardiology software packages. To perform this quantification, the 3-dimensional perfusion distribution is mapped to a standard template to eliminate the variability in LV size and shape between patients. The perfusion distribution is extracted from each LV short axis in the form of circumferential count profiles. The standard template is the 2-dimensional polar map (Fig. 1) that maps these count profiles into concentric rings from the base of the left ventricle (outer ring) to the apex (center of polar map) (4). Polar map distributions are computed in this manner for both the stress and the rest images from which estimates of stressinduced perfusion abnormalities can be identified and then further classified as ischemic or scar, using the rest map. The perfusion 


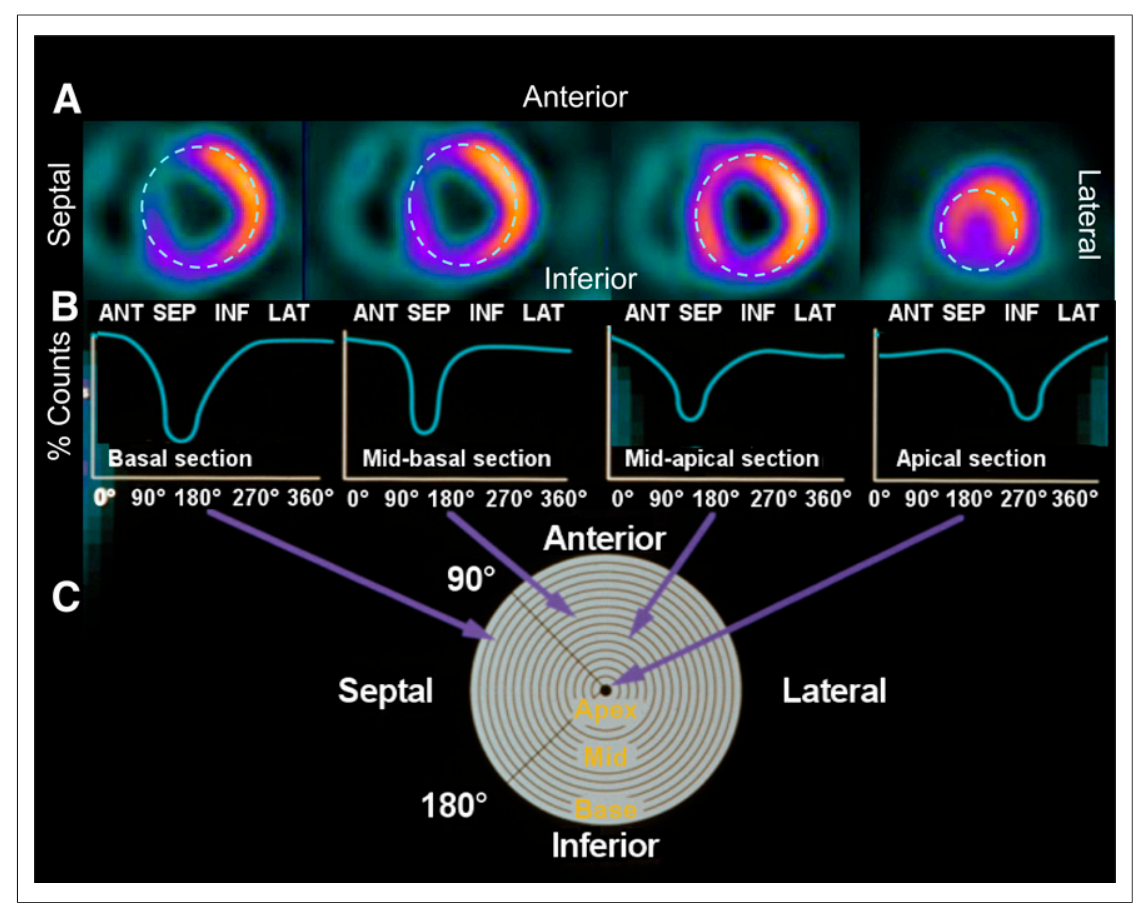

FIGURE 1. Method for polar map representation of LV myocardial perfusion distribution. (A) Circumferential count profiles are extracted from each short-axis slice from apex to base, depicted here as dashed circles (only 4 shown). (B) Circumferential profiles extracted from each LV short-axis slice plotted as normalized percentage counts extracted vs. angle around short axis for patient with hypoperfused septum. (C) Mapping of individual count profiles into rings, creating polar map.

SD of $5 \%$, one could use 2 SDs as the threshold for a mild defect $(70 \%=80 \%-2 \times 5 \%), 4$ SDs for a moderate defect $(60 \%)$, and 6 SDs for a severe defect $(50 \%)$. This methodology is still the standard for perfusion quantification (Fig. 2) (5). It has the advantage of increased accuracy over uniform thresholds because it can account for attenuation effects when the studies are not corrected for attenuation, which is the norm for SPECT MPI.

Normal Perfusion Databases. Regional attenuation effects require the use of a normal database comprising human subjects with body habitus that matches test patients. For this reason, databases from North America, where the mean body mass index is around 30, should not be used in countries where the mean body mass index is significantly lower, for example, in Japan, where the mean body mass index is $22(6)$. Other factors that influence attenuation effects are imaging position (supine, prone, upright) and photon energy; this means that matched databases should be used. The reconstruction algorithm also influences the estimated perfusion distribution, with the degree of smoothness dependent on the algorithm, filter, and number of iterations. One of the advantages of iterative reconstruction is that photon attenuation can be mod-

intensity in the most normal region within the LV is then used to normalize the polar map distribution to $100 \%$. This produces a relative perfusion distribution, with each sector's intensity being a percentage value relative to the uptake in the most normal myocardial region.

With the relative perfusion map, one can use simple thresholds to identify regions of mild (e.g., $<70 \%$ ), moderate (e.g., $<60 \%$ ), and severe (e.g., $<50 \%$ ) hypoperfusion. This methodology assumes that the normal perfusion distribution is uniform throughout the LV myocardium. Unfortunately, there are several processes that introduce nonuniformities in the measured data-the most notable being photon attenuation - that result in reduction or misplacement of photons, thereby altering the measured distribution from the true perfusion distribution. The most prominent attenuation artifacts are breast artifacts, in which the soft tissue of the breast causes a decrease in counts in the anteroseptal region of the map, and diaphragmatic artifacts, in which the soft tissue of the diaphragm or torso causes a decrease in counts in the inferolateral region of the map.

Relative Versus Database Quantification. Photon attenuation is very dependent on body habitus and tissue density, which means that it can vary significantly between patients. To measure the regional effects on the perfusion distribution, polar map distributions are typically acquired from a population comprising age-matched patients with a low $(\leq 5 \%)$ pretest likelihood of coronary artery disease (CAD). By averaging a large number (e.g., $>20$ ) of these distributions, estimates of the mean and its SD can be found for each sector in the polar map. A set of polar maps compiled in this manner is referred to as a normal perfusion database. Since the mean and SD are now estimated at each map sector, sector-specific thresholds based on a predefined number of SDs can be used. As an example, if a sector has a mean of $80 \%$ and an eled and corrected for in the reconstruction process. When the proper modeling and corrections are applied, the measured distribution is a close match to the true perfusion distribution. In this case, databases can be used interchangeably between sex, patient populations,

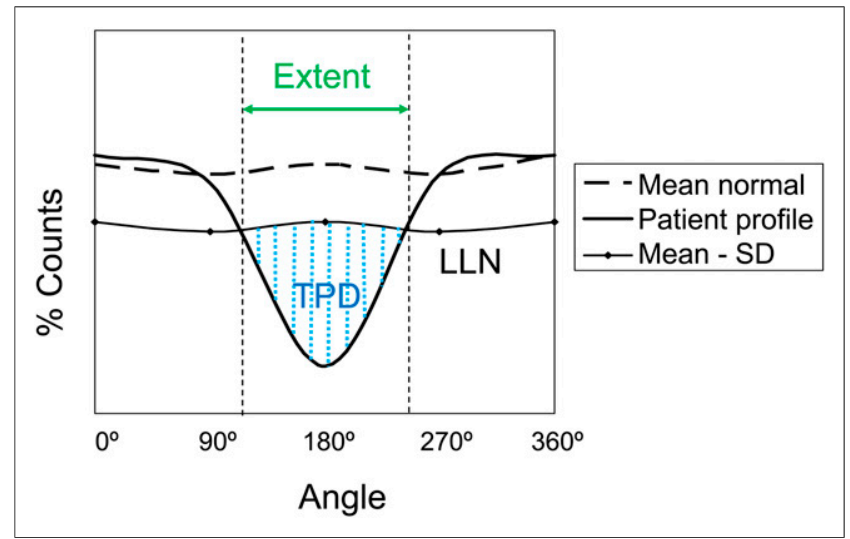

FIGURE 2. Methods for detecting and measuring degree of hypoperfusion. Plot depicts how circumferential count profile is tested for abnormality. Patient's normalized count profile (solid line) is compared against lower limit of normal (LLN) profile calculated as mean normal count response profile minus set number of SDs (usually 2-2.5). Extent of defect is given by angular range of count profile falling below LLN. Severity of deficit may be measured as sum of SD below mean normal profile for all abnormal angular samples. Total perfusion deficit (TPD) is marker of defect severity similar to SSS but measured for each sampled voxel, where each sample is scored from 0 (normal) to 4 (no uptake). Normal polar map generates TPD of 0 , and maximally abnormal polar map (no myocardial uptake) would result in TPD of $100 \%$. 
photon energy, and imaging position. This is more commonly seen in PET cardiac imaging.

The latest generations of dedicated cardiac SPECT cameras and advanced iterative reconstruction software methods (7-11) have enabled meaningful reductions in imaging time and radiopharmaceutical doses (12-16). However, if the reconstruction parameters are not adjusted for increased noise in the reduced-dose/time images, it may be necessary to use normal databases that are "countmatched" to avoid bias induced by the noise mismatch between the normal database and the reduced-dose/time images (15-17). Another factor that affects the normal perfusion distribution patterns is camera technology; Figure 3 illustrates examples of the mean normal perfusion polar map differences in regional relative perfusion between SPECT and PET normal patterns, standard SPECT versus cadmium-zinc-telluride imaging, and attenuation correction versus studies not corrected for attenuation.

Segmental Perfusion Scoring. Visually identifying the location and severity of a perfusion abnormality was standardized to the 17-segment model in 2002, providing a simple template to score the severity ( 0 to 4 : normal to absent) of the perfusion for the entire left ventricle (18). With the quantitative methods previously described using a normal database, thresholds can be mapped to each discrete severity score so that 17 -segment regional scoring maps can be automatically derived. Segmental scoring is done for both the stress and the rest studies, providing a regional representation of lesion location, severity, and changes from stress to rest.

The segmental summed stress score (SSS) and summed rest score have been shown to provide significant incremental prognostic value for nuclear cardiology (19). Using the summed scores, one can derive the percentage of the left ventricle $(\% \mathrm{LV})$ at risk as a ratio of SSS and the maximum summed value $[(68=17$ segments $\times 4$ maximum segmental score) $\times 100 \%$ ], which is a severity-weighted estimate of total defect size. Figure 4 illustrates how an SSS of 18 is converted to $26 \%$ of the left ventricle hypoperfused. Since quantitative polar maps have several hundred sectors, compared with the simplified 17 segments, a more accurate estimate of defect size can be performed directly with the polar map using the continuous severity score and is most commonly referred to as the total perfusion deficit (20) (Fig. 2). The automation of total perfusion deficit and its severity-weighted

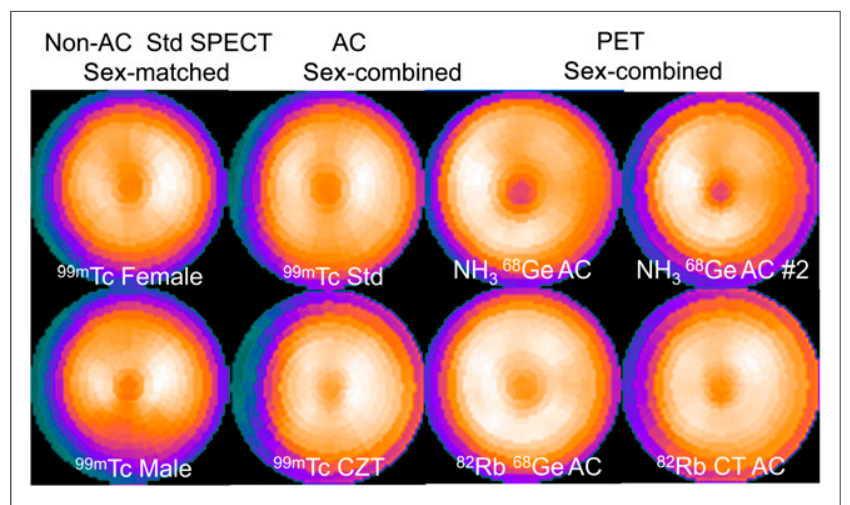

FIGURE 3. Comparison of different normal perfusion polar map patterns. Each of the 8 polar maps shown was generated from different patients with low likelihood of CAD as mean normal polar map relative response. Each normal pattern visually differs from the others. For example, attenuation-corrected $(\mathrm{AC})$ normal pattern shows increased relative counts in inferior wall, compared with normal polar map from noncorrected perfusion studies. $\mathrm{CZT}=$ cadmium zinc telluride; $\mathrm{Std}=$ standard SPECT.
$\% \mathrm{LV}$ at risk provides one more quantitative measure to be used in nuclear cardiology for the detection and characterization of CAD. Table 1 lists established quantitative measures of LV perfusion and their threshold criteria for abnormality.

\section{Reversibility/lschemic Burden}

Reversibility. The term reversibility refers to the extent and magnitude of improved perfusion when the myocardium is at rest, compared with the myocardial hypoperfusion at peak stress. The general approach is to generate a reversibility polar map distribution calculated by normalizing the same region in the resting distribution to the most normal region in the stress distribution. Once normalized, the normalized stress distribution (expressed as 0\%-100\%) is subtracted from the normalized resting distribution and what remains are the areas of improved perfusion at rest, or the reversibility map. This myocardial reversibility distribution is then compared with normal limits to establish if the differences are statistically significant (Fig. 5) (21). In normally perfused myocardium, the mean normal reversibility is zero as no relative changes are expected. This approach has been validated in a multicenter trial (22). Although the degree of reversibility from MPI studies is due to relative changes in myocardial blood flow between stress and rest, it is commonly clinically considered the degree of ischemia. Direct imaging of myocardial ischemia can be achieved by imaging radiopharmaceuticals that are free fatty acid analogs and glucose analogs (23).

Another approach to quantifying reversibility is to use the summed difference score (SDS) (Fig. 4). The SDS is determined as the SSS minus the summed rest score. For purposes of risk stratification, the SDS is categorized as low (0-2), intermediate (3-7), or high $(>7)$ (24).

Ischemic Burden. A more contemporary approach to measuring the degree of LV myocardial ischemia is the concept of ischemic burden. Quantification of this parameter relies on the SDS scores assigned using the 17-segment model (Fig. 4). The SDS score is converted to a global \%LV ischemic burden by dividing the SDS by the maximum possible SDS score (a score of $4 \times 17$ segments $=$ 68 ) and multiplying it by $100 \%$. Figure 4 illustrates how an SDS of 13 is converted to a $19 \% \mathrm{LV}$ ischemic burden. The reason this score has become popularized are the influential studies demonstrating that a cutoff of $10 \% \mathrm{LV}$ ischemic burden differentiates those patients who would lower their risk of cardiac death after revascularization $(\geq 10 \%$ ) from those patients who would benefit more from medical therapy alone $(<10 \%)(25,26)$. These findings imply that there has to be enough LV myocardium that is ischemic for an invasive procedure such as revascularization to reduce the risk of cardiac death to the patient.

Some clinicians use the stress \%LV hypoperfused amount as a proxy for the \%LV ischemic burden by converting the SSS score to $\% \mathrm{LV}$ by dividing the SSS by 68 and multiplying by $100 \%$. This substitution of SSS for SDS assumes that the reduction in counts at stress is completely due to ischemia. This assumption is true only when it is known that the patient has not had a previous myocardial infarction and that any potential attenuation artifacts have been accounted for by either attenuation correction or a combination of supine and prone imaging when using SPECT.

\section{Infarct Size/Viability}

Infarct Size. Quantification of infarct size with SPECT MPI uses the rationale that viable myocardial segments exhibit uptake of the perfusion tracer at rest whereas nonviable segments do not. The algorithm searches for the maximal counts in the entire LV 


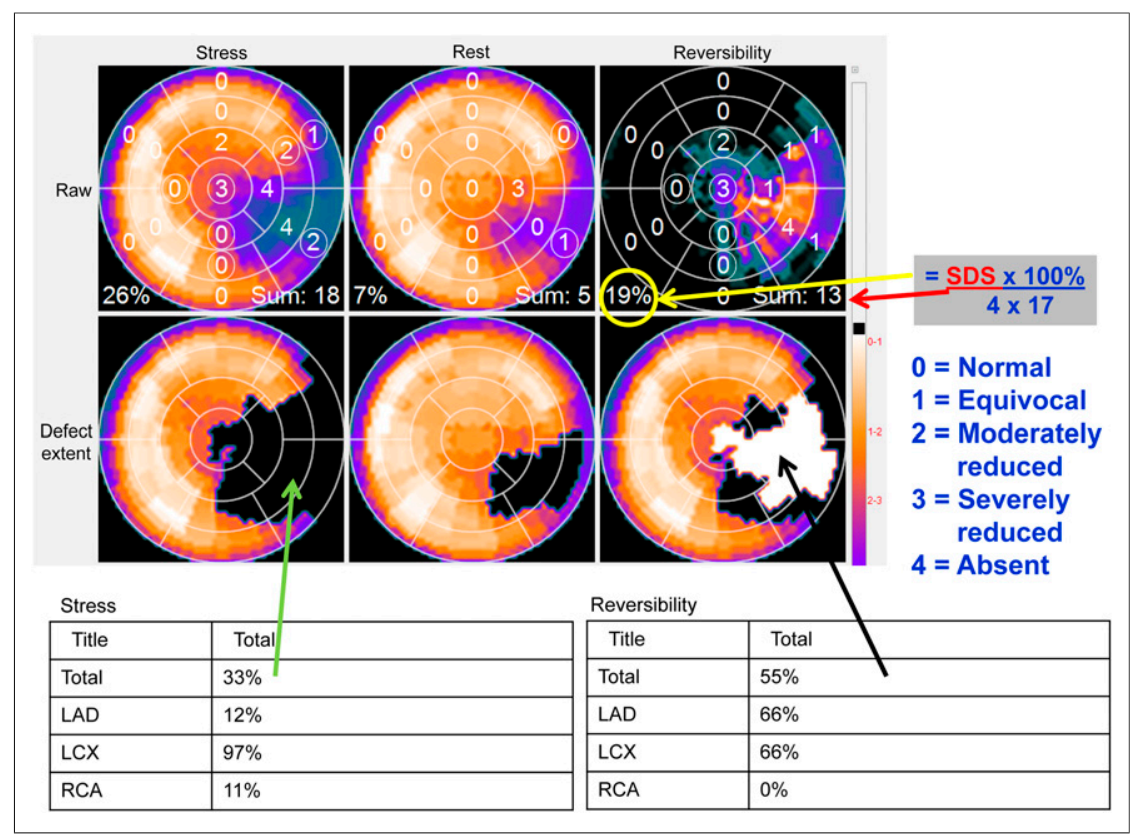

FIGURE 4. Methods for measuring degree of ischemic burden. Top row shows stress, rest, and reversibility polar maps scored (0-4) by computer algorithm using 17-segment model and their respective SSS, summed rest score, and SDS. SSS of 18 translates to $26 \%$ hypoperfused LV $[=100 \% \times 18 /(4 \times 17)]$. SDS of 13 translates to $19 \%$ LV ischemic burden, which is greater than $10 \%$ threshold for patient benefiting from revascularization and thus candidate for catheterization. Second row shows same polar maps after they have been compared with traditional normal databases explained in Figure 2. Tables below show that defect extent (blackout polar map) is $33 \%$ of LV and that $97 \%$ of left circumflex (LCX) vascular territory is abnormal, as well as $12 \%$ of left anterior descending (LAD) and $11 \%$ of right coronary artery (RCA) territories. Reversibility polar map (whiteout) shows that $55 \%$ of stress perfusion defect improves at rest.
Viability. Measurements of infarct size by SPECT MPI are widely used, particularly in clinical trials. Yet, it is commonly accepted that SPECT MPI underestimates myocardial viability, particularly in patients with severe LV dysfunction (31). Glucoseloaded ${ }^{18}$ F-FDG PET imaging, particularly when combined with resting perfusion imaging, remains the reference standard for measuring myocardial viability $(32,33)$.

Quantification of myocardial viability by combining resting MPI and a glucoseloaded ${ }^{18}$ F-FDG distribution relies on normalizing one distribution to the other before comparison. This normalization is done by first comparing the resting MPI distribution with an appropriate normal database. The hypoperfused areas can be identified by use of the normal database; the remaining normal areas are then used for normalization. The average count in the entire extent of normally perfused regions is computed for both the perfusion and the ${ }^{18}$ F-FDG studies. Then, the ${ }^{18} \mathrm{~F}-\mathrm{FDG}$ distribution is scaled so that its average in the normal region is equal to that in the perfusion study. After normalization, the normalized perfusion distribution is subtracted from the normalized metabolism $\left({ }^{18} \mathrm{~F}-\mathrm{FDG}\right)$ distribution. The difference between the distributions is expressed as a percentage of the normalized metabolism distribution. A threshold for this myocardial distribution and identifies as nonviable those myocardial segments that fall below a percentage of this maximal value, usually $45 \%-50 \%$ when using ${ }^{201} \mathrm{Tl}(27)$ and $60 \%$ when using for ${ }^{99 \mathrm{~m}} \mathrm{Tc}$ perfusion agents (28). This measurement has been validated with many other measures of infarct size (29). Alternatively, the normaldatabase approach can be used for determining the rest defect, and this method was found to correlate well with infarct size defined by delayed enhancement MRI (30). percentage difference can be set at any level (34), usually 5\%-10\%. Regions in the distribution that are above the threshold, and that have abnormal perfusion, are those that have relatively increased metabolism coexistent with relatively decreased perfusion. This region is known as the mismatch area. Any area above the threshold that is also abnormal for perfusion can be considered a mismatch consistent with myocardial hibernation and considered viable. If a myocardial region is statistically hypoperfused and the same region, in the

TABLE 1

Quantitative Measures of LV Perfusion

\begin{tabular}{|c|c|c|c|c|c|}
\hline & Parameter & Threshold & Measured & Reported & References \\
\hline \multirow[t]{3}{*}{ Global (LV) } & Defect extent (\%LV) & $3 \%-5 \%$ & Always & Frequently & $36-39$ \\
\hline & No. of SDs below mean normal & 2.5 & Always & Seldom & $36-39$ \\
\hline & Defect severity (\%LV) & $3 \%-5 \%$ & Always & Seldom & 24 \\
\hline \multirow[t]{3}{*}{ 17-segment model } & SSS & 4 & Always & Frequently & 24 \\
\hline & Ischemic burden (\%LV) & $10 \%-12 \%$ & Frequently & Frequently & 25 \\
\hline & Viability (\%LV) & $7 \%-20 \%$ & Frequently & Frequently & 35 \\
\hline Vascular territory & Defect extent (\% territory) & $3 \%-12 \%$ & Always & Frequently & $36-39$ \\
\hline Defect/territory & Stress-to-rest improvement & $2 \%-10 \%$ & Always & Frequently & $36-39$ \\
\hline 17-segment model & SDS & 2 & Always & Frequently & 26 \\
\hline
\end{tabular}

Ranges in thresholds are due to differences in protocols or software used. Potential for measuring the listed quantitative parameters depends on the specific software used, which also includes version number associated with age of algorithms. 


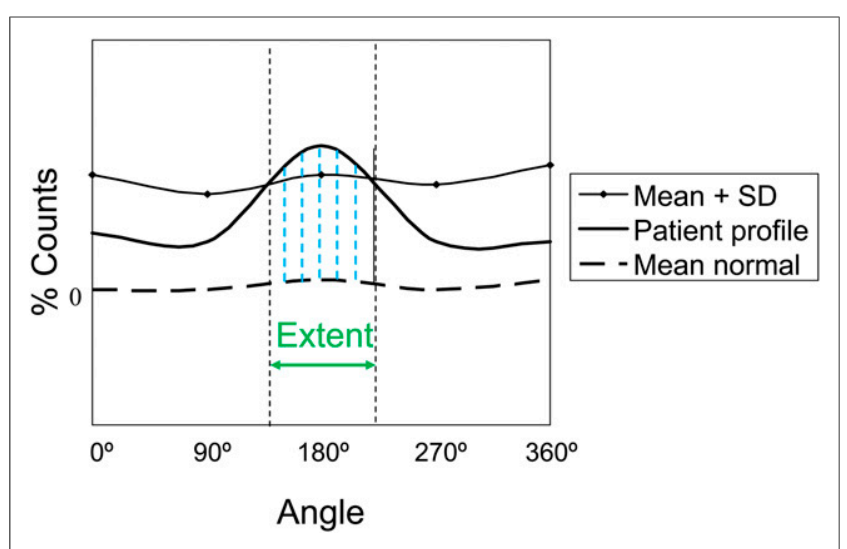

FIGURE 5. Method for detecting and measuring degree of defect reversibility. Plot depicts how circumferential reversibility profile is tested for improvement. Reversibility patient profile is generated by subtracting normalized stress count profile from resting count profile. Angular range that improves in relative perfusion from stress to rest is shown by increasing reversibility above zero. Mean normal reversibility profile hovers around zero since in healthy patients no relative perfusion change is expected between stress and rest. Angular extent of significant reversibility is depicted as portion of reversibility profile that jumps above upper limit of normal reversibility given by mean normal plus statistically determined number of SDs.

${ }^{18}$ F-FDG distribution, does not exceed the expected threshold for improvement, then these regions are considered to be matched and not viable. The extent of both viable and nonviable myocardium is quantified as \%LV and, if the myocardial mass is computed, may be expressed in terms of grams of myocardial tissue. Although the presence of viable myocardium implies an increased likelihood of functional improvement with revascularization $(32,33)$, there is currently a lack of consensus on the quantitative threshold of viable extent that would predict improvement, varying in different studies between $7 \%$ and $20 \%$ (35). This concept closely follows the concept of $\% \mathrm{LV}$ ischemic burden explained above except that it is usually applied to patients with more advanced heart disease, such as heart failure.

\section{Global LV Function}

Global Functional Measurement. It is strongly recommended that electrocardiography gating be performed with all SPECT and PET protocols to allow assessment of multiple ventricular function parameters in addition to perfusion variables. Automatic computer-based methods quantify indices of global function, including LV ejection fraction (LVEF), end-diastolic volume, end-systolic volume, transient ischemic dilation (TID), myocardial mass, and diastolic function at stress and rest.

Quantitation of ventricular function and volumes with gated SPECT MPI can be performed by a variety of algorithms. The most common approaches are based on the automatic detection of endocardial and epicardial surface points across the cardiac cycle in 3 dimensions and may also consider the principle of constant mass across the cardiac cycle for improved robustness of the contour detection (Fig. 6) (36-39). In several independent studies (40-43) of LVEF obtained by SPECT MPI, the agreement between gated MPI and other standard measurements of LVEF has been shown to be very good to excellent, particularly when correlating LVEFs along a continuum from high to low values. Although LVEFs measured by different quantitative algorithms correlate highly, the specific cutoffs for abnormal LVEF may be method-dependent (43). For some methods, the normal threshold for the global LVEF measured by gated SPECT MPI images can be lower than that measured by other imaging modalities or by different algorithms. Unless the method uses Fourier temporal filtering (44), one of the reasons for the apparently lower LVEF is the use of only 8 gating intervals for binning the electrocardiography data. Recently, 16-frame gating has become more common in SPECT MPI, reducing the underestimation of LVEF. Normal limits for LVEF and LV volumes may depend on the sex of the patient, probably because of differences in absolute LV size in relation to SPECT image resolution, resulting in underestimation of end-systolic volume in patients with small hearts (45), particularly when end-systolic volume is less than $15 \mathrm{~mL}$. Table 2 lists established quantitative measures of LV function and their threshold criteria for abnormality.

Diastolic Function. Another group of parameters representing global ventricular function that can be obtained from MPI is diastolic function indices, in particular peak filling rate (PFR), defined as the greatest filling rate in early diastole. Diastolic function is traditionally assessed by echocardiography and gated blood-pool studies. PFR corresponds to the peak value of the first derivative of the diastolic portion of the gated frame curve (46) and can be normalized to end-diastolic volume, providing a clinically intuitive index. Additionally, time to PFR in milliseconds can be reported. Abnormality of LV diastolic function could be an early sign of CAD, congestive heart failure, and other cardiac conditions. The assessment of diastolic function has been shown to be feasible with gated SPECT MPI (47). When 16-frame gating is used, normal limits for PFR and time to PFR are similar to those reported with gated blood-pool studies. Agreement between SPECT and MRI in both PFR and time to PFR has been demonstrated when 32-frame gating is used for SPECT (48). Global time to PFR was shown to be less sensitive than regional parameters (49). Assessment of global diastolic function is not yet widely used, and the clinical value of MPI-derived diastolic function requires further evaluation.

Nonperfusion Parameters. In addition to perfusion defects, several nonperfusion characteristics can be observed with SPECT MPI, including mass (50), shape (51), and TID of the LV (52). Since contours of the LV are derived from gated and ungated scans, an estimate of the myocardial mass can be based on the volume between computed endo- and epicardial surfaces (53). Determination of endo- and epicardial surfaces requires that these 3-dimensional surfaces be generated by a computer algorithm such as with edge detection of the myocardium. The reproducibility of interobserver measurements for LV mass obtained from poststress MPI has been shown to be very high (54). LV mass derived by SPECT MPI also correlated with mass derived by CT; nevertheless, limits of agreement were wide, with lower values overestimated and higher values underestimated (54).

TID. Quantitative TID, in particular, has attracted clinical attention as an additional marker of high-risk disease derived by MPI. TID is usually calculated from the nongated rest and stress images as the ratio of LV cavity volume at stress divided by the volume at rest; however, the ratio can also be calculated from end-diastolic images obtained by gated SPECT (55). TID is considered present when the LV cavity appears to increase in the poststress images compared with rest images (52). This phenomenon may represent either apparent cavity dilation or subendocardial ischemia, which may explain why TID may be seen for several 


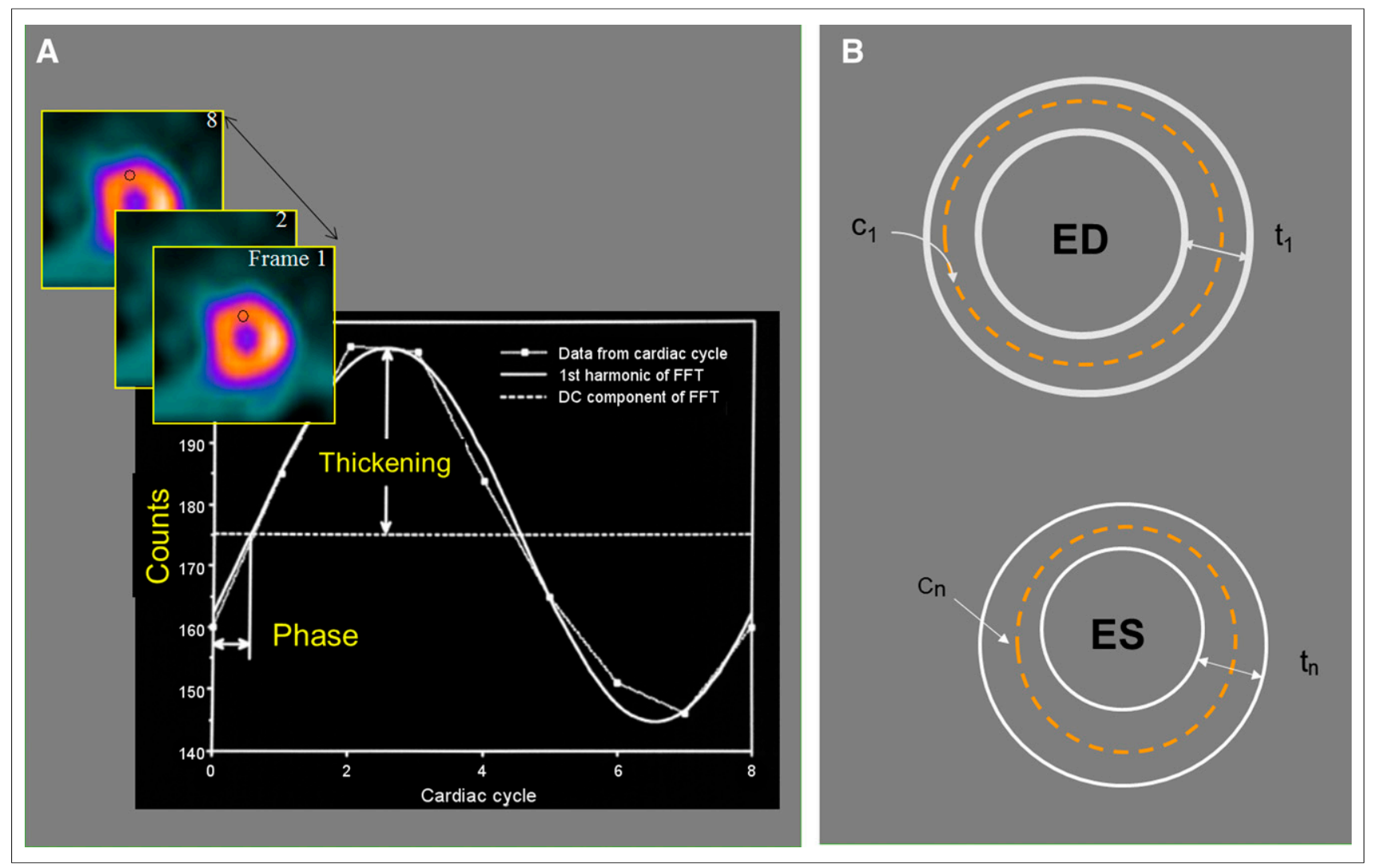

FIGURE 6. Method of detecting endocardial and epicardial LV boundaries for measuring global and regional function. (A) Maximal-count 3dimensional sampling (c) of same LV myocardial region throughout cardiac cycle can be plotted as function of time (frame number) to extract percentage thickening throughout cardiac cycle. (B) Once end-diastolic (ED) 3-dimensional thickness (t) is determined (measured or assumed) to define endocardial and epicardial contours, change in maximal counts from ED is usually used to model how these contours move throughout cardiac cycle. These contours at ED and at end-systole (ES) are used to measure global LVEF, end-diastolic volume, and end-systolic volume. These same contour movements throughout cardiac cycle are used to measure mass, wall motion, and wall thickening, as well as diastolic function parameters. DC $=$ average thickening for segment; FFT $=$ Fast Fourier Transform.

hours after stress, whereas functional abnormalities may no longer be present (Fig. 7). It appears that pharmacologically induced TID has prognostic implications similar to TID caused by exercise (56). Quantitative TID assessment has been shown to improve the detection of severe CAD when combined with perfusion assessment (55). The abnormality threshold for TID depends on the camera type, stress type, and imaging protocol used (57). A comprehensive comparison of abnormality thresholds for 8 different protocols (both exercise- and vasodilator-based) using data from 5 imaging centers from a large multinational registry, REFINESPECT (58), in 1,672 patients with a low likelihood of CAD and normal scan findings has been reported (58). The TID abnormality thresholds varied from 1.18 to 1.52 between various clinical protocols currently used $(58,59)$. Protocols such as ${ }^{82} \mathrm{Rb}$, for which the stress perfusion images are acquired closer to peak stress, tend to yield a lower normal TID due to a reduction in the apparent LV chamber size at stress. TID upper limits have also been shown to be 1.15 for ${ }^{82} \mathrm{Rb}$ PET MPI studies (60), lower than those for SPECT. This variability of TID across protocols underscores the importance of matching the TID limits to the specific MPI protocol used while integrating quantitative TID assessment to the clinical practice.

Calcium Score. The use of hybrid SPECT/CT cameras allows for attenuation correction and for a more comprehensive assessment of CAD. Noncontrast CT images can be used for SPECT attenuation correction and, when electrocardiography-gated, for coronary artery calcium scoring $(61,62)$. Both of these attributes have been shown to increase diagnostic performance (62). Because coronary calcium is pathognomonic of $\mathrm{CAD}$, it can reclassify the risk and improve diagnosis in patients without known CAD who have normal findings on myocardial perfusion SPECT imaging. Moreover, the absence of coronary artery calcium (score of 0 ) is associated with a very low probability of future cardiovascular events (62). This information should be used to considerably lower the patient's posttest probability of $\mathrm{CAD}$ and to increase the diagnostic confidence of the physician in excluding obstructive disease. Laboratories that measure the calcium score frequently report it.

\section{Regional LV Function}

Myocardial Thickening. Measurement of myocardial thickening is based on the observation that because of the limited spatial resolution of our imaging cameras compared with the myocardial thickness, a linear change in maximal counts in myocardial segments occurs as a direct response to a change in thickness (63) Thus, relative thickening is measured as a percent increase in maximal counts for each myocardial segment throughout the cardiac cycle. Even though gated MPI studies routinely use 8-16 frames per cardiac cycle, the temporal resolution of thickening is improved by the use 
TABLE 2

Quantitative Measures of LV Function

\begin{tabular}{|c|c|c|c|c|c|}
\hline \multirow[b]{2}{*}{ Parameter } & \multicolumn{2}{|c|}{ Threshold abnormal } & \multirow[b]{2}{*}{ Measured } & \multirow[b]{2}{*}{ Reported } & \multirow[b]{2}{*}{ References } \\
\hline & Males & Females & & & \\
\hline \multicolumn{6}{|l|}{ Systolic/global } \\
\hline LVEF (\%) & $<43-52$ & $<51-60$ & Always & Always & $36-39,45$ \\
\hline End-diastolic volume ( $\mathrm{mL})$ & $>149-197$ & $>102-122$ & Always & Frequently & $36-39,45$ \\
\hline End-systolic volume (mL) & $>70-82$ & $>42-46$ & Always & Frequently & $36-39,45$ \\
\hline LV mass (g) & $>208$ & $>158$ & Frequently & Seldom & 36 \\
\hline Summed thickening score & $>3$ & $>3$ & Frequently & Seldom & 64 \\
\hline Summed wall motion score & $>3$ & $>3$ & Frequently & Seldom & 64 \\
\hline TID & $1.14-1.36$ & $1.14-1.36$ & Always & Frequently & $55-59,60$ \\
\hline \multicolumn{6}{|l|}{ Diastolic/global } \\
\hline PFR (end-diastolic volume/s) & $<1.7$ & & Frequently & Seldom & 46 \\
\hline Time to PFR (ms) & $>208$ & & Frequently & Seldom & 46 \\
\hline
\end{tabular}

Note that potential for measuring the listed quantitative parameters depends on the specific software used, which also includes version number associated with age of algorithms.

of the Fourier transform (44). This mathematic step may be thought of as fitting to a sine wave the discrete counts from the same myocardial region in each of the 8 frames. Segmental thickening is then better approximated as the amplitude of the sine wave that was fitted to the counts from those regions. Quantitative programs display polar maps of regional (relative) thickening, often comparing it to a database of normal regional thickening. Average thickening by approximately more than $40 \%$ at the apex and more than $25 \%$ in the rest of the left ventricle is considered normal.

Myocardial Wall Motion. For measurement of myocardial endocardial wall motion, determination of the 3-dimensional LV endocardium is required. Determination of the endocardial boundary may be done using edge detection techniques, assumptions as to myocardial thickness, calibration to a myocardial phantom, or combinations of these.
Application of edge detection techniques to MPI studies is limited by the low spatial resolution of the nuclear imaging systems. The accuracy of this approach may be improved by calibrating against myocardial phantoms of known thickness. The other methods require a determination of the absolute myocardial thickness. The thickening method measures relative thickening rather than absolute thickness. One approach uses the myocardial location of the maximal count (corrected for scatter) to determine the midpoint of the myocardial wall segment $(37,44)$. The assumption is made that at end-diastole the LV myocardial thickness is $1 \mathrm{~cm}$, that is, $5 \mathrm{~mm}$ on either side of the midpoint. This defines the 3-dimensional endocardial and epicardial surfaces. It then uses the regional change in counts from the enddiastolic frame to determine the change in endocardial/epicardial surface location. For example, if at end-systole the myocardial counts in a segment double, the thickness will also double from 1 to $2 \mathrm{~cm}$.

Myocardial Wall Thickening Versus Wall Motion. The use of myocardial wall thickening to express regional ventricular function has gained preferential status. The rationale is that the myocardium must thicken in order for the endocardium to have a meaningful wall motion. The endocardium may move without corresponding thickening, but this is due to either tethering to a region that is thickening or the torque of the heart. Neither of these 2 phenomena contribute to the stroke volume. Fully automated scoring of regional SPECT MPI LV wall motion and thickening can outperform experienced observers in the detection of CAD (64).

FIGURE 7. Method for detecting TID. (Top) TID due to change in LV cavity size dilated during stress as compared with smaller cavity size at rest. Blue ellipses depict hypothetical cavity size (in actuality done in 3 dimensions) to demonstrate difference between TID and transient subendocardial ischemia (TSI) and are not meant to be true detection of endocardial LV border. (Bottom) TID due to subendocardial ischemia during stress that normalizes at rest. Actual size of LV epicardium does not change from stress to rest. This is perhaps better named transient subendocardial ischemia. Cavity volumes at stress and rest are calculated in 3 dimensions from endocardial contours determined by computer algorithm.

\section{Diagnostic Performance of \\ Established Applications} tification of SPECT and PET MPI has been extensively tested for diagnostic accuracy versus invasive angiography. MPI worldwide
Diagnostic Performance. Relative quan- 
is performed mainly by SPECT ( $>90 \%)$. In a large clinical study of 995 patients, automated quantitative SPECT analysis was compared with visual analysis (65). A fully automated computer analysis of either attenuation-corrected or non-attenuation-corrected SPECT MPI data has been shown to be equivalent per patient and potentially superior for per-vessel analysis, when compared with expert analysis for the detection of stenoses of at least $70 \%$ based on angiographic criteria (66). The advanced methods allowing fully automated relative quantification were primarily optimized and validated for SPECT MPI. There are, however, equivalent tools available for relative perfusion quantification with PET. For PET, smaller studies quantitating MPI were performed also demonstrating high diagnostic accuracy (67).

Automation. The main advantage of computer-based relative quantification of perfusion over visual analysis is objectivity and reproducibility. Even expert physicians' reading styles may differ, resulting in discrepancy in the subjective findings. Diagnostic agreement between 2 expert observers was found to be limited, with wide margins of error for the segmental summed scores (68). The reproducibility of software analysis is much better. In a study comparing visual and quantitative reproducibility of parameters obtained from repeated scans after a single injection, the quantification of perfusion deficit was found to have about half the variability of the intraobserver segmental scoring (same observer reading repeated in $3 \mathrm{wk}$ ) (69). Constant improvements in automatic processing allow reduced operator intervention and, consequently, further reproducibility improvements of the quantitative results, such as when all the available studies (stress, rest, gated, ungated) from the same patient study are processed in the same session

It has been demonstrated (70) that the repeatability coefficients of automatic perfusion quantification have been reduced to $2.6 \%$ and the failure rate for perfusion image analysis has been reduced to less than $1 \%$. Techniques have been developed for the automatic flagging of the possible LV segmentation errors (71). Such methods allow processing of MPI images with minimal operator supervision, as was recently demonstrated in a large prognostic study (72).

Attenuation Correction and 2-Position Imaging. Automatic quantification can be performed either with or without AC (73). The analysis of most MPI AC studies shows significant improvement over non-AC studies in the specificity of detecting CAD (74). However, some groups (75) have attained only a modest improvement in diagnostic accuracy (by $\sim 3 \%$ ) for perfusion quantification of attenuation-corrected images, as compared with noncorrected data. The use of AC for quantification of myocardial perfusion may eliminate the need for sex-specific databases (76). If attenuation maps are not available for correction, it is possible to use tomograms acquired from multiple patient positions (supine, prone, upright) to assess if a decrease in image counts is related to attenuation artifacts, since patient position alters attenuation artifacts (77). Quantitative techniques have been developed to estimate the size of perfusion defects simultaneously from 2 image positions-supine and prone acquisitions on conventional cameras $(78,79)$ and upright and supine views on dedicated cardiac cameras $(80)$. These quantitative techniques demonstrate improved prediction of obstructive disease by mitigating attenuation artifacts, similarly to attenuation-corrected analysis.

Quantitative Risk Prediction. The overall accuracy and precision of automatic analysis are critical for diagnostic purposes. The quantitative analysis could potentially be used directly for prognostic risk assessment $(66,72,81)$. However, ultimately, the prediction of the relative benefit after therapy could be performed by quantitative rather than visual estimates of ischemia. Nevertheless, the validation studies to date of the ischemic defect size threshold for guiding therapy did not use the quantitatively determined $\% \mathrm{LV}$ ischemic burden but rather relied on subjective visual scoring of the SDS converted to the \% LV ischemic burden (26).

\section{CONCLUSION}

Quantitative clinical software tools in nuclear cardiology allow the objectification and standardization of measurements of cardiac perfusion, function, metabolism, innervation, and inflammationa major strength of this imaging technique. Established tools are used clinically on a daily basis on most cardiac patients undergoing nuclear cardiology procedures to complement the diagnosticians' acumen and to assist in study interpretation, clinical management, and therapy guidance.

\section{REFERENCES}

1. Iskandrian AE, Garcia EV, eds. Nuclear Cardiac Imaging. 5th ed. New York, NY: Oxford University Press; 2016

2. Dorbala S, Ananthasubramaniam K, Armstrong IS, et al. Single Photon Emission Computed Tomography (SPECT) myocardial perfusion imaging guidelines: instrumentation, acquisition, processing, and interpretation. J Nucl Cardiol. 2018;25: 1784-1846.

3. Dilsizian V, Bacharach SL, Beanlands RS, et al. ASNC imaging guidelines/SNMMI procedure standard for positron emission tomography (PET) nuclear cardiology procedures. J Nucl Cardiol. 2016;23:1187-1226.

4. Garcia EV, Van Train K, Maddahi J, et al. Quantification of rotational thallium-201 myocardial tomography. J Nucl Med. 1985;26:17-26.

5. Van Train KF, Areeda J, Garcia EV, et al. Quantitative same-day rest-stress technetium-99m-sestamibi SPECT: definition and validation of stress normal limits and criteria for abnormality. $J$ Nucl Med. 1993;34:1494-1502.

6. Nakajima K, Matsumoto N, Kasai T, Matsuo S, Kiso K, Okuda K. Normal values and standardization of parameters in nuclear cardiology: Japanese Society of Nuclear Medicine working group database. Ann Nucl Med. 2016;30:188-199.

7. Gambhir SS, Berman DS, Ziffer J, et al. A novel high-sensitivity rapid-acquisition single-photon cardiac imaging camera. J Nucl Med. 2009;50:635-643.

8. Esteves FP, Raggi P, Folks RD, et al. Novel solid-state-detector dedicated cardiac camera for fast myocardial perfusion imaging: multicenter comparison with standard dual detector cameras. J Nucl Cardiol. 2009;16:927-934.

9. Bai C, Conwell R, Kindem J, et al. Phantom evaluation of a cardiac SPECT/VCT system that uses a common set of solid-state detectors for both emission and transmission scans. J Nucl Cardiol. 2010;17:459-469.

10. Bocher M, Blevis IM, Tsukerman L, Shrem Y, Kovalski G, Volokh L. A fast cardiac gamma camera with dynamic SPECT capabilities: design, system validation and future potential. Eur J Nucl Med Mol Imaging. 2010;37:1887-1902.

11. Imbert L, Poussier S, Franken PR, et al. Compared performance of high-sensitivity cameras dedicated to myocardial perfusion SPECT: a comprehensive analysis of phantom and human images. J Nucl Med. 2012;53:1897-1903.

12. Duvall WL, Croft LB, Godiwala T, Ginsberg E, George T, Henzlova MJ. Reduced isotope dose with rapid SPECT MPI imaging: initial experience with a CZT SPECT camera. J Nucl Cardiol. 2010;17:1009-1014.

13. Lecchi M, Martinelli I, Zoccarato O, Maioli C, Lucignani G, Del Sole A. Comparative analysis of full-time, half-time, and quarter-time myocardial ECG-gated SPECT quantification in normal-weight and overweight patients. J Nucl Cardiol. 2017;24:876-887.

14. Lyon MC, Foster C, Ding X, et al. Dose reduction in half-time myocardial perfusion SPECT-CT with multifocal collimation. J Nucl Cardiol. 2016;23:657-667.

15. Nakazato R, Berman DS, Hayes SW, et al. Myocardial perfusion imaging with a solid-state camera: simulation of a very low dose imaging protocol. J Nucl Med. 2013;54:373-379.

16. Sharir T, Pinskiy M, Pardes A, et al. Comparison of the diagnostic accuracies of very low stress-dose with standard-dose myocardial perfusion imaging: automated quantification of one-day, stress-first SPECT using a CZT camera. J Nucl Cardiol. 2016;23:11-20.

17. Wetzl M, Sanders JC, Kuwert T, Ritt P. Effect of reduced photon count levels and choice of normal data on semi-automated image assessment in cardiac SPECT. J Nucl Cardiol. April 13, 2018 [Epub ahead of print]. 
18. Cerqueira MD, Weissman NJ, Dilsizian V, et al. Standardized myocardial segmentation and nomenclature for tomographic imaging of the heart: a statement for healthcare professionals from the Cardiac Imaging Committee of the Council on Clinical Cardiology of the American Heart Association. Circulation. 2002;105: 539-542.

19. Berman DS, Hachamovitch R, Kiat H, et al. Incremental value of prognostic testing in patients with known or suspected ischemic heart disease: a basis for optimal utilization of exercise technetium- $99 \mathrm{~m}$ sestamibi myocardial perfusion single-photon emission computed tomography. J Am Coll Cardiol. 1995;26:639-647.

20. Slomka PJ, Nishina H, Berman DS, et al. Automated quantification of myocardial perfusion SPECT using simplified normal limits. J Nucl Cardiol. 2005;12:66-77.

21. Klein JL, Garcia EV, DePuey EG, et al. Reversibility bullseye: a new polar bull's-eye map to quantify reversibility of stress induced SPECT Tl-201 myocardial perfusion defects. J Nucl Med. 1990;31:1240-1246.

22. Garcia EV, DePuey EG, Sonnemaker RE, et al. Quantification of the reversibility of stress induced SPECT thallium-201 myocardial perfusion defects: a multicenter trial using bull's-eye polar maps and standard normal limits. J Nucl Med. 1990;31: 1761-1765.

23. Jain D, He ZH, Lele V, Aronow WS. Direct myocardial ischemia imaging: a new cardiovascular nuclear imaging paradigm. Clin Cardiol. 2015;38:124-130.

24. Berman DS, Kang X, Van Train KF, et al. Comparative prognostic value of automatic quantitative analysis versus semiquantitative visual analysis of exercise myocardial perfusion single-photon emission computed tomography. J Am Coll Cardiol. 1998;32:1987-1995.

25. Hachamovitch R, Rozanski A, Shaw LJ, et al. Impact of ischaemia and scar on the therapeutic benefit derived from myocardial revascularization vs. medical therapy among patients undergoing stress-rest myocardial perfusion scintigraphy. Eur Heart J. 2011;32:1012-1024.

26. Hachamovitch R, Hayes SW, Friedman JD, Cohen I, Berman D. Comparison of the short-term survival benefit associated with revascularization compared with medical therapy in patients with no prior coronary artery disease undergoing stress myocardial perfusion single photon emission computed tomography. Circulation. 2003;107:2900-2907.

27. Tamaki S, Nakajima H, Murakami T, et al. Estimation of infarct size by myocardial emission computed tomography with thallium-201 and its relation to creatine kinase-MB release after myocardial infarction in man. Circulation. 1982;66: 994-1001.

28. Gibbons RJ, Verani MS, Behrenbeck T, et al. Feasibility of tomographic ${ }^{99 \mathrm{~m}} \mathrm{Tc}-$ hexalis-2-methoxy2-methylpropyl-isonitrile imaging for the assessment of myocardial area at risk and the effect of treatment in acute myocardial infarction. Circulation. 1989;80:1277-1286.

29. Christian TF. The use of perfusion imaging in acute myocardial infarction: applications for clinical trials and clinical care. J Nucl Cardiol. 1995;2:423-436.

30. Slomka PJ, Fieno D, Thomson L, et al. Automatic detection and size quantification of infarcts by myocardial perfusion SPECT: clinical validation by delayedenhancement MRI. J Nucl Med. 2005;46:728-735.

31. Dilsizian V, Arrighi JA, Diodati JG, et al. Myocardial viability in patients with chronic coronary artery disease: comparison of ${ }^{99 \mathrm{~m}} \mathrm{Tc}$-sestamibi with thallium reinjection and $\left[{ }^{18} \mathrm{~F}\right]$ fluorodeoxyglucose. Circulation. 1994;89:578-587.

32. Tillisch J, Brunken R, Marshall R, et al. Reversibility of cardiac wall-motion abnormalities predicted by positron tomography. N Engl J Med. 1986;314:884-888.

33. Schelbert HR, Beanlands R, Bengel F, et al. PET myocardial perfusion and glucose metabolism imaging: part 2-guidelines for interpretation and reporting. J Nucl Cardiol. 2003;10:557-571.

34. Santana CA, Faber TL, Soler-Peter M, et al. Prognostic performance of quantitative PET tools for stratification of patients with ischemic cardiomyopathy undergoing myocardial viability assessment. Nucl Med Commun. 2008;29:970-981.

35. Allman KC. Noninvasive assessment myocardial viability: current status and future directions. J Nucl Cardiol. 2013;20:618-637.

36. Ficaro EP, Lee BC, Kritzman JN, Corbett JR. Corridor4DM: the Michigan method for quantitative nuclear cardiology. J Nucl Cardiol. 2007;14:455-465.

37. Garcia EV, Faber TL, Cooke CD, Folks RD, Chen J, Santana C. The increasing role of quantification in clinical nuclear cardiology: the Emory approach. $\mathrm{J} \mathrm{Nucl}$ Cardiol. 2007;14:420-432.

38. Germano G, Kavanagh PB, Slomka PJ, Van Kriekinge SD, Pollard G, Berman DS. Quantitation in gated perfusion SPECT imaging: the Cedars-Sinai approach. J Nucl Cardiol. 2007;14:433-454.

39. Slomka PJ, Germano G, Berman DS. Gated SPECT MPI processing and quantitation. In: Iskandrian AE, Garcia EV. eds. Nuclear Cardiac Imaging. 5th ed. New York, NY: Oxford University Press; 2016:109-136.

40. Stegger L, Lipke CSA, Kies P, et al. Quantification of left ventricular volumes and ejection fraction from gated ${ }^{99 \mathrm{~m}} \mathrm{Tc}-\mathrm{MIBI}$ SPECT: validation of an elastic surface model approach in comparison to cardiac magnetic resonance imaging, 4D-MSPECT and QGS. Eur J Nucl Med Mol Imaging. 2007;34:900-909.
41. Soneson H, Hedeer F, Arévalo C, et al. Development and validation of a new automatic algorithm for quantification of left ventricular volumes and function in gated myocardial perfusion SPECT using cardiac magnetic resonance as reference standard. J Nucl Cardiol. 2011;18:874-885.

42. Schaefer WM, Lipke CSA, Standke D, et al. Quantification of left ventricular volumes and ejection fraction from gated ${ }^{99 \mathrm{~m}} \mathrm{Tc}-\mathrm{MIBI}$ SPECT: MRI validation and comparison of the Emory Cardiac Tool Box with QGS and 4D-MSPECT. J Nucl Med. 2005;46:1256-1263.

43. Schaefer WM, Lipke CS, Nowak B, et al. Validation of QGS and 4D-MSPECT for quantification of left ventricular volumes and ejection fraction from gated ${ }^{18}$ F-FDG PET: comparison with cardiac MRI. J Nucl Med. 2004;45:74-79.

44. Cooke CD, Garcia EV, Cullom SJ, Faber TL, Pettigrew RI. Determining the accuracy of calculating systolic wall thickening using a fast Fourier transform approximation: a simulation study based on canine and patient data. J Nucl Med. 1994;35:1185-1192.

45. Sharir T, Kang X, Germano G, et al. Prognostic value of poststress left ventricular volume and ejection fraction by gated myocardial perfusion SPECT in women and men: gender-related differences in normal limits and outcomes. J Nucl Cardiol. 2006;13:495-506.

46. Akincioglu C, Berman DS, Nishina $\mathrm{H}$, et al. Assessment of diastolic function using 16-frame ${ }^{99 \mathrm{~m}} \mathrm{Tc}$-sestamibi gated myocardial perfusion SPECT: normal values. J Nucl Med. 2005;46:1102-1108.

47. Nakajima K, Taki J, Kawano M, et al. Diastolic dysfunction in patients with systemic sclerosis detected by gated myocardial perfusion SPECT: an early sign of cardiac involvement. J Nucl Med. 2001;42:183-188.

48. Kuroiwa Y, Nagamachi S, Miyati T, et al. The agreement of left ventricular function parameters between ${ }^{99 \mathrm{~m}} \mathrm{Tc}$-tetrofosmin gated myocardial SPECT and gated myocardial MRI. Ann Nucl Med. 2012;26:147-163.

49. Yamamoto A, Takahashi N, Abe K, Kobayashi Y, Tamai J, Munakata K. Regional left-ventricular diastolic wall motion assessed by a new program for ECG-gated myocardial perfusion SPECT in early-stage heart failure. J Nucl Cardiol. 2008;15: 375-382.

50. Gimelli A, Liga R, Magro S, et al. Evaluation of left ventricular mass on cadmium-zinc-telluride imaging: validation against cardiac magnetic resonance. J Nucl Cardiol. 2019;26:899-905.

51. Abidov A, Slomka PJ, Nishina H, et al. Left ventricular shape index assessed by gated stress myocardial perfusion SPECT: initial description of a new variable. J Nucl Cardiol. 2006;13:652-659.

52. Mazzanti M, Germano G, Kiat H, et al. Identification of severe and extensive coronary artery disease by automatic measurement of transient ischemic dilation of the left ventricle in dual-isotope myocardial perfusion SPECT. J Am Coll Cardiol. 1996;27:1612-1620.

53. Faber TL, Cooke CD, Folks RD, et al. Left ventricular function and perfusion from gated SPECT perfusion images: an integrated method. J Nucl Med. 1999;40: 650-659.

54. Okwuosa TM, Hampole CV, Ali J, Williams KA. Left ventricular mass from gated SPECT myocardial perfusion imaging: comparison with cardiac computed tomography. J Nucl Cardiol. 2009;16:775-783.

55. $\mathrm{Xu} \mathrm{Y,} \mathrm{Arsanjani} \mathrm{R,} \mathrm{Clond} \mathrm{M,} \mathrm{et} \mathrm{al.} \mathrm{Transient} \mathrm{ischemic} \mathrm{dilation} \mathrm{for} \mathrm{coronary}$ artery disease in quantitative analysis of same-day sestamibi myocardial perfusion SPECT. J Nucl Cardiol. 2012;19:465-473.

56. Abidov A, Bax JJ, Hayes SW, et al. Transient ischemic dilation ratio of the left ventricle is a significant predictor of future cardiac events in patients with otherwise normal myocardial perfusion SPECT. J Am Coll Cardiol. 2003;42: 1818-1825.

57. Abidov A, Germano G, Berman DS. Transient ischemic dilation ratio: a universal high-risk diagnostic marker in myocardial perfusion imaging. J Nucl Cardiol. 2007; 14:497-500.

58. Slomka PJ, Betancur J, Liang JX, et al. Rationale and design of the REgistry of Fast Myocardial Perfusion Imaging with NExt generation SPECT (REFINE SPECT). J Nucl Cardiol. June 19, 2018 [Epub ahead of print].

59. Hu LH, Sharir T, Miller RJH, et al. Upper reference limits of transient ischemic dilation ratio for different protocols on new-generation cadmium zinc telluride cameras: a report from REFINE SPECT registry. J Nucl Cardiol. May 13, 2019 [Epub ahead of print].

60. Shi H, Santana CA, Rivero A, et al. Normal values and prospective validation of transient ischemic dilation index in Rb-82 PET myocardial perfusion imaging. Nucl Med Commun. 2007;28:859-863.

61. Esteves FP, Santana CA, Raggi P, Garcia EV. Myocardial perfusion SPECT/CT: The added value of CT imaging. In: Iskandrian AE, Garcia EV, eds. Atlas of Nuclear Cardiology: Companion to Braunwald's Heart Disease. Philadelphia, PA: Saunders; 2012:390-416.

62. Sarwar A, Shaw LJ, Shapiro MD, et al. Diagnostic and prognostic value of absence of coronary artery calcification. JACC Cardiovasc Imaging. 2009;2:675-688. 
63. Galt JR, Garcia EV, Robbins W. Effects of myocardial wall thickness on SPECT quantification. IEEE Trans Med Imaging. 1990;9:144-150.

64. Slomka PJ, Berman DS, Xu Y, et al. Fully automated wall motion and thickening scoring system for myocardial perfusion SPECT: method development and validation in large population. J Nucl Cardiol. 2012;19:291-302.

65. Arsanjani R, Xu Y, Hayes SW, et al. Comparison of fully automated computer analysis and visual scoring for detection of coronary artery disease from myocardial perfusion SPECT in a large population. J Nucl Med. 2013;54:221-228.

66. Nakazato R, Berman DS, Gransar H, et al. Prognostic value of quantitative highspeed myocardial perfusion imaging. J Nucl Cardiol. 2012;19:1113-1123.

67. Santana CA, Folks RD, Garcia EV, et al. Quantitative Rb-82 PET/CT development and validation of myocardial perfusion database. J Nucl Med 2007;48:1122-1128.

68. Slomka PJ, Dey D, Sitek A, Motwani M, Berman DS, Germano G. Cardiac imaging: working towards fully-automated machine analysis \& interpretation. Expert Rev Med Devices. 2017;14:197-212.

69. Xu Y, Fish M, Gerlach J, et al. Combined quantitative analysis of attenuation corrected and non-corrected myocardial perfusion SPECT: method development and clinical validation. J Nucl Cardiol. 2010;17:591-599.

70. Germano G, Kavanagh PB, Ruddy TD, et al. "Same-patient processing" for multiple cardiac SPECT studies. 2. Improving quantification repeatability. J Nucl Cardiol. 2016;23:1442-1453.

71. Xu Y, Kavanagh P, Fish M, et al. Automated quality control for segmentation of myocardial perfusion SPECT. J Nucl Med. 2009;50:1418-1426.

72. Motwani M, Leslie WD, Goertzen AL, et al. Fully automated analysis of attenuation-corrected SPECT for the long-term prediction of acute myocardial infarction. J Nucl Cardiol. 2018;25:1353-1360.

73. Ficaro EP, Fessler JA, Shreve PD, Kritzman JN, Rose PA, Corbett JR. Simultaneous transmission/emission myocardial perfusion tomography: diagnostic accuracy of attenuation-corrected ${ }^{99 \mathrm{~m}} \mathrm{Tc}$-sestamibi single-photon emission computed tomography. Circulation. 1996;93:463-473.

74. Garcia EV. Quantitative nuclear cardiology: we are almost there! J Nucl Cardiol. 2012;19:424-437.

75. Slomka PJ, Fish MB, Lorenzo S, et al. Simplified normal limits and automated quantitative assessment for attenuation-corrected myocardial perfusion SPECT. J Nucl Cardiol. 2006;13:642-651.

76. Grossman GB, Garcia EV, Bateman TM, et al. Quantitative Tc-99m sestamibi attenuation-corrected SPECT: development and multicenter trial validation of myocardial perfusion stress gender-independent normal database in an obese population. J Nucl Cardiol. 2004;11:263-272.

77. Hayes SW, De Lorenzo A, Hachamovitch R, et al. Prognostic implications of combined prone and supine acquisitions in patients with equivocal or abnormal supine myocardial perfusion SPECT. J Nucl Med. 2003;44:1633-1640.

78. Nishina H, Slomka PJ, Abidov A, et al. Combined supine and prone quantitative myocardial perfusion SPECT: method development and clinical validation in patients with no known coronary artery disease. J Nucl Med. 2006;47: 51-58.

79. Slomka PJ, Nishina H, Abidov A, et al. Combined quantitative supine-prone myocardial perfusion SPECT improves detection of coronary artery disease and normalcy rates in women. $J$ Nucl Cardiol. 2007;14:44-52.

80. Nakazato R, Tamarappoo BK, Kang X, et al. Quantitative upright-supine highspeed SPECT myocardial perfusion imaging for detection of coronary artery disease: correlation with invasive coronary angiography. J Nucl Med. 2010;51: 1724-1731.

81. Leslie WD, Tully SA, Yogendran MS, Ward LM, Nour KA, Metge CJ. Prognostic value of automated quantification of Tc-99m-sestamibi myocardial perfusion imaging. J Nucl Med. 2005;46:204-211. 